Borna Fardsadegh, Hamideh Vaghari, Roya Mohammad-Jafari, Yahya Najian

and Hoda Jafarizadeh-Malmiri*

\title{
Biosynthesis, characterization and antimicrobial activities assessment of fabricated selenium nanoparticles using Pelargonium zonale leaf extract
}

https://doi.org/10.1515/gps-2018-0060

Received March 8, 2018; accepted May 25, 2018; previously published online September 7, 2018

Abstract: The present study focuses on the biogenic synthesis of selenium nanoparticles (Se NPs) using Pelargonium zonale leaf extract under microwave irradiation. Response surface methodology was used to evaluate the effects of the synthesis parameters, namely amounts of the leaf extract $(0.5-2.5 \mathrm{ml})$ and amounts of the $10 \mathrm{~mm}$ sodium selenite solutions (15-65 ml), at constant microwave heating ( $4 \mathrm{~min}$ ), on the concentration and particle size of the fabricated Se NPs, optimize the synthesis conditions and verify the generated models and the procedures. The obtained results indicated that Se NPs with preferable attributes of mean particle size $(50 \mathrm{~nm})$, zeta potential $(-24.6 \mathrm{mV})$, absorbance [34.6\% absorbance units (a.u.)] and broad absorption peak (319 $\mathrm{nm}$ ) were formed at the optimum synthesis conditions including amounts of $1.48 \mathrm{ml}$ and $15 \mathrm{ml}$ Pelargonium leaf extract and sodium selenite solution, respectively. The antibacterial activities of the synthesized Se NPs against Escherichia coli and Staphylococcus aureus indicated that the created NPs had higher antibacterial activities toward the Gram-positive bacteria. Furthermore, the synthesized Se NPs indicated higher antifungal activities against Colletotrichum coccodes and Penicillium digitatum.

Keywords: biological synthesis; microwave irradiation; Pelargonium zonale; response surface methodology; selenium nanoparticles.

\footnotetext{
*Corresponding author: Hoda Jafarizadeh-Malmiri, Faculty of Chemical Engineering, Sahand University of Technology, East Azarbaijan, Tabriz 51335-1996, Iran, e-mail: h_jafarizadeh@sut.ac.ir, h_jafarizadeh@yahoo.com Borna Fardsadegh: Faculty of Chemical Engineering, Sahand University of Technology, East Azarbaijan, Tabriz, Iran Hamideh Vaghari: Faculty of Chemical Engineering, Sahand University of Technology, East Azarbaijan, Tabriz, Iran; and Research and Development Department, Najian Herbal Group, East Azarbaijan, Tabriz, Iran

Roya Mohammad-Jafari and Yahya Najian: Research and Development Department, Najian Herbal Group, East Azarbaijan, Tabriz, Iran
}

\section{Introduction}

Selenium as an essential trace element for the human body and animals is a non-metal element with low toxicity [1]. Selenium has high antioxidant activity and is present in the form of selenoproteins in animals in which it acts as cofactor in vital enzymatic reactions [2, 3]. Furthermore, selenium has been widely used in pharmaceuticals and medicine due to its anticarcinogenic, muscle functioning and positive effect on thyroid metabolism [3, 4]. Selenium nanoparticles (Se NPs) indicate higher antitumor activity as compared to its organic and inorganic components in the bulk form [5]. Therefore, Se NPs as a novel therapeutic and anticancer agent has gained more attention by researchers, in the last decade $[5,6]$.

Like other metal and non-metal NPs, Se NPs have attracted more interest because of their excellent bioavailability, antioxidant activity, anticancer property, and lower toxicity $[2,7,8]$. Several studies revealed the mentioned beneficial properties of the redness synthesized of Se NPs and their higher adsorptive ability interaction with numerous functional groups (i.e. $\mathrm{N}-\mathrm{H}, \mathrm{C}-\mathrm{O}, \mathrm{COO}$ and $\mathrm{C}-\mathrm{N}$ ) in human and animal bodies [7].

Several physical and chemical techniques, based on the utilization of the chemical compounds, have been developed to synthesize Se NPs; the residual of these chemicals limits the applications of the formed Se NPs in the pharmaceutical and medicinal areas $[8,9]$. Therefore, emerging efforts and studies have been greatly interested in the biological synthesis of Se NPs, resulting in the development of a non-toxic and eco-friendly synthesis approach $[9,10]$. Use of the plant extracts in the biological synthesis of NPs is easier and more cost effectives as compared to those biological synthesis methods utilizing microorganisms (i.e. bacteria and fungi) and enzymes [11].

Pelargonium/Geranium belongs to the family Geraniaceae and its leaves have a pleasant lemon aroma which is widely used as a flavoring agent in soaps, fruit desserts, ice cream, cake and jellies formulations [12,13]. Furthermore, its leaves contain tannin, flavonoids, sesquiterpenes, phenolic acid, cinnamic acid, coumarin and monoterpenes which can act as both reducing and stabilizing 
agents in synthesis of NPs [14, 15]. The rate of biological NPs synthesis is moderate as compared to that of chemical synthesis methods. However, combination of the biological methods with the physical heating techniques can effectively accelerate the rate of NPs fabrication. Among the conventional heating methods, microwave irradiation has several advantages [16, 17]. Microwave radiation can be easily absorbed by the mixture solution containing the ions salt and reducing agents, and localized superheating occurs, resulting in fast and efficient heating. Several studies have indicated that the green synthesized silver and gold NPs under microwave irradiation had minimum particle size and particle size distributions, and maximum stability $[16,18]$.

The main aims of the present study were to: (i) evaluate the potential application of the Pelargonium zonale leaves extract (PLE) in synthesis of the Se NPs, (ii) optimize the biological synthesis conditions of the Se NPs to achieve Se NPs with suitable physico-chemical attributes including minimum particle size and maximum zeta potential value (stability), and (iii) assay antibacterial and antifungal activities of the synthesized Se NPs against Gram-positive and Gram-negative bacteria species.

\section{Materials and methods}

\subsection{Materials}

Pelargonium zonale leaves and sodium selenite $\left(\mathrm{Na}_{2} \mathrm{SeO}_{3}\right)$ (purity $>99 \%$ ), as a natural source of bioreducing and stabilizing agents, and a selenium precursor, were provided from the local market (Tabriz, Iran) and Merck Company (Merck Co., Darmstadt, Germany), respectively. Escherichia coli (PPTCC 1270) and Staphylococcus aureus (PTCC 1112) were purchased from the microbial Persian type culture collection (PTCC, Tehran, Iran). Nutrient agar and potato dextrose agar (PDA) were purchased from Himedia (Himedia Co., Mumbai, India) and Oxoid (Oxoid Ltd., Hampshire, UK), respectively. Colletotrichum coccodes and Penicillium digitatum were isolated from local spoiled tomatoes and oranges, respectively.

\subsection{Pelargonium leaf extract preparation}

In order to prepare the PLE, the prepared leaves of Pelargonium zonale of similar size, shape and color, were washed and shade-dried during a week at ambient conditions (temperature of $30^{\circ} \mathrm{C}$ and relative humidity of $27 \%$ ). The dried leaves were then powdered by domestic miller (MX-GX1521; Panasonic, Tokyo, Japan) and $2 \mathrm{~g}$ of the prepared powder was added into the $100 \mathrm{ml}$ of boiling deionized double distilled water for $5 \mathrm{~min}$. PLE was finally obtained by filtration of the boiled solution through Whatman No. 40 filter paper.

\subsection{Se NPs synthesis using PLE}

Previous studies which completed the synthesis of Se NPs utilized $10 \mathrm{mM} \mathrm{Na}_{2} \mathrm{SeO}_{3}$ as selenium solution $[5,19,20]$. Therefore, $0.263 \mathrm{~g}$ of the selenium salt was dissolved in $100 \mathrm{ml}$ of deionized double distilled water to obtain $10 \mathrm{~mm}$ colorless selenium solution and several defined amounts of $\mathrm{Na}_{2} \mathrm{SeO}_{3}$ solution (15-65 ml) and PLE (0.5-2.5 ml) were mixed and then exposed to the microwave radiation using a domestic microwave oven (MG2312w, LG Co., Seoul, South Korea) for $4 \mathrm{~min}$ and at constant power of $800 \mathrm{~W}$.

\subsection{PLE characteristics assay}

A Fourier transform-infrared (FT-IR) instrument (Shimadzu 8400S spectrometer, Kyoto, Japan) coupled with $\mathrm{KBr}$ pellets $\left(4000-400 \mathrm{~cm}^{-1}\right.$ region) was utilized to monitor and detect the functional groups of PLE. These groups are related to the main presented reducing and stabilizing bioactive compounds in the PLE. The $\mathrm{pH}$ of PLE was measured using a pH meter (DELTA 320; Delta, Shanghai, China).

\subsection{Assessment of the fabricated Se NPs characteristics}

UV-Vis spectrophotometry (Jenway UV-Vis spectrophotometer 6705, Stone, UK) was used to confirm the formation of the Se NPs based on their surface plasmon resonance (SPR) signal. Due to the SPR signal of the NPs, the mixture solution containing the formed Se NPs indicated the broad absorption peak $\left(\lambda_{\max }\right)$ in the wavelength ranged 270-350 nm [19, 20]. The measured absorbance of the solution containing the formed NPs could be correlated to the concentration of the synthesized Se NPs. By contrast, the higher absorbance unit (\% a.u.) of the mixture solution revealed that Se NPs with higher concentrations were synthesized and vice versa [15]. Furthermore, by formation of the Se NPs, the color of the mixture solution containing selenium ions converted from pale yellow into dark red for the solution containing the formed Se NPs [19, 21, 22].

Particle size, polydispersity index (PDI), particle size distribution and zeta potential values of the synthesized Se NPs were evaluated and recorded by a dynamic light scattering (DLS) analyzer (Malvern instruments, Zetasizer Nano ZS, Worcestershire, UK). For the morphology studies of the synthesized Se NPs, transmission electron microscopy (TEM CM120, Philips, Amsterdam, Netherlands) with an acceleration voltage of $120 \mathrm{kV}$, was used. The exact value of the fabricated Se NPs was also calculated and determined by TEM.

\subsection{Antimicrobial activities of the synthesized Se NPs}

Antibacterial activities of the synthesized Se NPs against Gram-positive (S. aureus) and Gram-negative (E. coli) bacteria were evaluated using the well diffusion method described by Torabfam and JafarizadehMalmiri [23]. The antibacterial assay was performed in incubation conditions of the $37^{\circ} \mathrm{C}$ for $24 \mathrm{~h}$.

The antifungal activities of the fabricated Se NPs were evaluated against $C$. coccodes and $P$. digitatum using an inhibition in radial mycelia growth method described by Mohammadlou et al. [15]. 
The antifungal assay was completed in incubation conditions of $26^{\circ} \mathrm{C}$ during 7 days. In order to isolate the studied fungi strains, pieces of the spoiled tomato and orange peels, which were inoculated with the $C$. coccodes and $P$. digitatum, respectively, were separated and put into the center of the plates into which PDA was poured. The provided plates were then incubated at $26^{\circ} \mathrm{C}$ for 1 week and after that a $5 \mathrm{~mm}$ disc (in diameter) of the growth fungi were provided to place on the surface of the plates containing PDA and synthesized Se NPs, PDA and PLE, and PDA as a control sample.

\subsection{Statistical analysis}

Central composite design with five replications of the center point, and response surface methodology (RSM) based on a second-order polynomial model, were used to design experiments and evaluate the effects of the synthesis parameters, namely amounts of PLE ( $\mathrm{X}_{1}$, $\mathrm{ml})$ and $\mathrm{Na}_{2} \mathrm{SeO}_{3}$ solution $\left(\mathrm{X}_{2}, \mathrm{ml}\right)$, on the $\lambda_{\max }(\mathrm{nm})$ and absorbance (\% a.u.) values of the solutions containing formed Se NPs [16, 24]. As compared to other statistical techniques, RSM has several advantages including decreasing the experimental runs, and costs and evaluation of the interaction effects of the independent variables on the response variables $[25,26]$. Coefficient of determination $\left(R^{2}\right)$, adjusting the coefficient of determination $\left(R^{2}\right.$-adj) and lack of fit ( $p$-value) were evaluated for the suitability of the generated models. In addition, the significance determination of the resulted models was studied using analysis of variance in term of $p$-value at the $95 \%$ confidence interval for the level of the significance. Three-dimensional surface plots and two-dimensional contour plots were established to well observation of the interaction of the synthesis parameters and the optimum region of the response variables, respectively [15].

Based on the synthesis of Se NPs with minimum $\lambda_{\text {max }}$ (nm) and maximum absorbance (\% a.u.), a graphical optimization using an overlaid contour plot was obtained and a numerical optimization was carried out to achieve the strict optimum levels of the selected fabrication variables in the defined ranges. Validity of the generated models based on RSM to predict the optimum synthesis conditions, was evaluated using three additional approval tests for the synthesized Se NPs at obtained optimum conditions [27, 28]. The experimental design, data analysis optimization and verification procedures were performed using Minitab software (Minitab Inc., v. 16, PA, USA).

\section{Results and discussion}

\subsection{Specification of the PLE}

The main functional groups existing in the PLE are shown in Figure 1. As can be seen in the FT-IR spectra of the PLE (Figure 1), there were four highlighted peaks centered at $3452.5 \mathrm{~cm}^{-1}, 2066 \mathrm{~cm}^{-1}, 1636.5 \mathrm{~cm}^{-1}$ and $662.8 \mathrm{~cm}^{-1}$. The two main peaks were detected at $3452.5 \mathrm{~cm}^{-1}$ and $1636.5 \mathrm{~cm}^{-1}$, which were related to the stretching vibrations of hydroxyl and amide groups, respectively. Hydroxyl groups have a key role in the reduction of selenium ions to their element

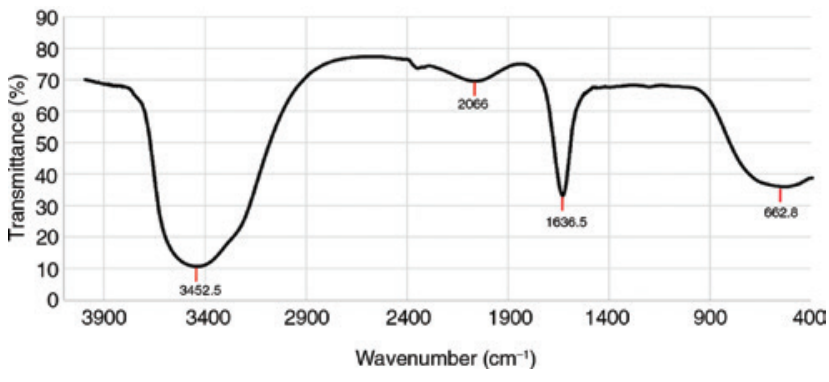

Figure 1: Fourier transform-infrared (FT-IR) spectrum of Pelargonium zonale leaves extract (PLE).

and can be found in the structure of the tannins, flavonoids, phenolic acid, cinnamic acid, coumarin, monoterpenes, and sesquiterpenes which are presented in the PLE [15]. By contrast, amide groups have a main role in the stabilizing of the formed Se NPs, and these exist in the structure of the proteins and enzymes of the PLE $[15,16]$. The $\mathrm{pH}$ of the provided PLE was approximately 5.5, indicating that the PLE is an acidic solution.

\subsection{Evaluation of the fitted models as a function of the amounts of PLE and $\mathrm{Na}_{2} \mathrm{SeO}_{3}$ solution}

According to the design of experiments, and achieved experimental values for the $\lambda_{\max }(\mathrm{nm})$ and absorbance (\% a.u.) (Table 1), the second polynomial models based

Table 1: Experimental runs according to the central composite design (CCD) for selenium nanoparticles (Se NPs) synthesis.

\begin{tabular}{|c|c|c|c|c|c|c|}
\hline \multirow[t]{2}{*}{$\begin{array}{l}\text { Sample } \\
\text { number }\end{array}$} & \multirow[t]{2}{*}{$\begin{array}{r}\text { Amount of } \\
\text { PLE (ml) }\end{array}$} & \multirow[t]{2}{*}{$\begin{array}{r}\text { Amount of } \\
\mathrm{Na}_{2} \mathrm{SeO}_{3}(\mathrm{ml})\end{array}$} & \multicolumn{2}{|c|}{$\lambda_{\max }(\mathrm{nm})$} & \multicolumn{2}{|c|}{$\begin{array}{r}\text { Absorbance } \\
\left(\% \text { a.u } u^{\mathrm{a}}\right)\end{array}$} \\
\hline & & & $\operatorname{Exp}^{b}$ & Pre $^{c}$ & $\operatorname{Exp}^{b}$ & Pre \\
\hline 1 & 0.79 & 32.22 & 319 & 318.93 & 20.01 & 2.19 \\
\hline 2 & 0.50 & 40.00 & 321 & 320.89 & 1.93 & 2.22 \\
\hline 3 & 0.79 & 57.67 & 322 & 323.35 & 1.65 & 0.74 \\
\hline 4 & 2.21 & 57.67 & 322 & 322.56 & 5.57 & 4.18 \\
\hline 5 & 2.50 & 40.00 & 323 & 322.60 & 9.09 & 10.05 \\
\hline 6 & 1.50 & 15.00 & 319 & 319.04 & 35.58 & 35.47 \\
\hline 7 & 1.50 & 40.00 & 324 & 323.20 & 5.22 & 5.49 \\
\hline 8 & 1.50 & 40.00 & 322 & 323.20 & 5.54 & 5.49 \\
\hline 9 & 1.50 & 40.00 & 323 & 323.20 & 5.74 & 5.49 \\
\hline 10 & 1.50 & 40.00 & 324 & 323.20 & 5.50 & 5.49 \\
\hline 11 & 1.50 & 40.00 & 323 & 323.20 & 5.47 & 5.49 \\
\hline 12 & 1.50 & 65.00 & 323 & 322.45 & 5.08 & 5.07 \\
\hline 13 & 2.20 & 22.32 & 321 & 321.14 & 28.12 & 27.77 \\
\hline
\end{tabular}

${ }^{\mathrm{a} A b s o r b a n c e}$ unit. ${ }^{\mathrm{b}}$ Experimental values of studied responses. 'Predicted values of studied responses. 
on Eq. (1) were generated as a function of the linear $\left(\mathrm{X}_{\mathrm{i}}\right)$, quadratic $\left(\mathrm{X}_{\mathrm{ii}}\right)$ and interaction $\left(\mathrm{X}_{\mathrm{ij}}\right)$ of the amounts of PLE $\left(\mathrm{X}_{1}, \mathrm{ml}\right)$ and $\mathrm{Na}_{2} \mathrm{SeO}_{3}$ solution $\left(\mathrm{X}_{2}, \mathrm{ml}\right)$. $\mathrm{Y}$ is the response variable:

$$
Y=\beta_{0}+\beta_{1} X_{1}+\beta_{2} X_{2}+\beta_{11} X_{1}^{2}+\beta_{22} X_{2}^{2}+\beta_{12} X_{1} X_{2}
$$

The regression coefficients of the generated models with their $\mathrm{R}^{2}, \mathrm{R}^{2}$-adj and $\mathrm{p}$-values of the lack of fit are given in Table 2. As can be seen in this table, the higher values of the $R^{2}(>0.88) R^{2}$-adj (>0.80) and high p-values of the lack of fit $(p>0.05)$ confirmed the adequate fitness of the generated models. Table 3 presents the $\mathrm{p}$-values of all the terms of the models. As can be observed in this table, the interaction of the synthesis parameters had non-significant effects on the $\lambda_{\text {max }}(\mathrm{nm})$ of the solution containing Se NPs. By contrast, the quadratic effect of the amounts of PLE $\left(\mathrm{X}_{11}, \mathrm{ml}\right)$ had insignificant effects on the absorbance of the resulting solutions.

The $\lambda_{\max }$ of the solutions containing Se NPs was changed from $319 \mathrm{~nm}$ to $324 \mathrm{~nm}$. It is noted that the

Table 2: Regression coefficients, $R^{2}, R^{2}$-adj, and probability values for the fitted models.

\begin{tabular}{lrr}
\hline Regression coefficient & $\boldsymbol{\lambda}_{\max }(\mathrm{nm})$ & Absorbance (\% a.u.) \\
\hline$\beta_{0}$ (constant) & 307.254 & 58.2049 \\
$\beta_{1}$ (main effect) & 6.804 & 5.3259 \\
$\beta_{2}$ (main effect) & 0.442 & -2.3741 \\
$\beta_{11}$ (quadratic effect) & -1.450 & 0.6485 \\
$\beta_{22}$ (quadratic effect) & -0.004 & 0.0237 \\
$\beta_{12}$ (interaction effect) & -0.040 & -0.0839 \\
$R^{2}$ & 88.35 & 99.57 \\
R-adj & 80.02 & 99.25 \\
Lack of fit (p-value) & 0.736 & 0.100 \\
\hline
\end{tabular}

$\beta_{0}$ is constant and $\beta_{\mathrm{i}}, \beta_{\mathrm{ii}}$ and, $\beta_{\mathrm{ij}}$ are the linear, quadratic and interaction coefficients of the quadratic polynomial equation, respectively. 1 , pelargonium extract $(\mathrm{ml}) ; 2$, amount of $\mathrm{Na}_{2} \mathrm{SeO}_{3}(\mathrm{ml})$. a.u., Absorbance unit.

Table 3: $p$-Value of the regression coefficients in the obtained models.

\begin{tabular}{|c|c|c|c|c|c|}
\hline \multirow[t]{2}{*}{ Main effects } & \multicolumn{2}{|c|}{ Main effects } & \multicolumn{2}{|c|}{$\begin{array}{r}\text { Quadratic } \\
\text { effects }\end{array}$} & \multirow{2}{*}{$\begin{array}{r}\begin{array}{r}\text { Interacted } \\
\text { effects }\end{array} \\
\mathrm{X}_{12}\end{array}$} \\
\hline & $X_{1}$ & $x_{2}$ & $X_{11}$ & $X_{22}$ & \\
\hline \multicolumn{6}{|l|}{$\lambda_{\max }(\mathrm{nm})$} \\
\hline $\mathrm{p}$-Value & 0.013 & 0.001 & 0.034 & 0.003 & 0.213 \\
\hline \multicolumn{6}{|c|}{ Absorbance (\% a.u.) } \\
\hline p-Value & 0.001 & 0.000 & 0.562 & 0.000 & 0.002 \\
\hline
\end{tabular}

1, pelargonium extract (ml); 2, amount of $\mathrm{Na}_{2} \mathrm{SeO}_{3}(\mathrm{ml})$. a.u., Absorbance unit. particle size of the synthesized Se NPs can be exhibited in the $\lambda_{\text {max }}$ of them. It is identified that the higher $\lambda_{\max }$ values for the NPs are interrelated to their larger size [29]. The effects of the synthesis parameters on the $\lambda_{\max }$ of the synthesized Se NPs are shown in Figure 2A and B. As can be detected in Figure 2A, at any constant amount of PLE, by increasing the amount of $\mathrm{Na}_{2} \mathrm{SeO}_{3}$ solution the $\lambda_{\max }$ were increased. The same trend was also observed for increasing the amount of PLE at any constant amount of the $\mathrm{Na}_{2} \mathrm{SeO}_{3}$ solution. This indicated that the interaction of the synthesis variables had non-significant effects on the $\lambda_{\text {max }}$ of the solution containing Se NPs. The obtained result was reconfirmed by the obtained p-value $>0.05$ of the synthesis parameters interaction in Table 3. As can be seen in Figure $2 \mathrm{~B}$, minimum $\lambda_{\max }$ of the solution containing Se NPs could be obtained at the lower amounts of the $\mathrm{PLE}$ and $\mathrm{Na}_{2} \mathrm{SeO}_{3}$ solution.

As can be seen in Table 1, the absorbance (\% a.u.) values of the solutions containing formed Se NPs varied from 1.654 to 35.58 (\% a.u.). It is noted that the concentration of the synthesized Se NPs can be manifested in the absorbance of their solutions. It is identified that the higher absorbance for the NPs is correlated to their higher concentrations [29]. The effects of the studied fabrication variables on the absorbance of the solutions are indicated in Figure $2 \mathrm{C}$ and $\mathrm{D}$. As clearly detected in Figure 2C, at a constant amount of PLE, by increasing the amount of the $\mathrm{Na}_{2} \mathrm{SeO}_{3}$ solution the absorbance was decreased. This trend was observed throughout any amount of the PLE. At constant low and high amounts of the $\mathrm{Na}_{2} \mathrm{SeO}_{3}$ solution, by increasing the amount of PLE, different patterns were observed. The obtained results indicated that the fabrication parameters interaction had a significant $(\mathrm{p}<0.05)$ effect on the absorbance value of the solution containing fabricated Se NPs, which was in agreement with the obtained p-value for the interaction of the synthesis parameters (Table 3). Figure 2D, revealed that the maximum values for the absorbance of the solution containing Se NPs were obtained at higher amounts of PLE and lower amounts of the $\mathrm{Na}_{2} \mathrm{SeO}_{3}$ solution. The achieved result can be described by the point that, at high amounts of PLE, the concentrations of the bioreductant compounds existing in the PLE are high and these reducing agents can easily and rapidly reduce the presented selenium in the solution and convert them into their NPs, which caused an increase in the concentration of the synthesized Se NPs. The attained results were in line with findings of Mohammadlou et al. [15] and Eskandari et al. [17, 30]. Those indicated that in synthesis of the silver and gold NPs using PLE and mushroom extract, by increasing the amounts of the extracts, 

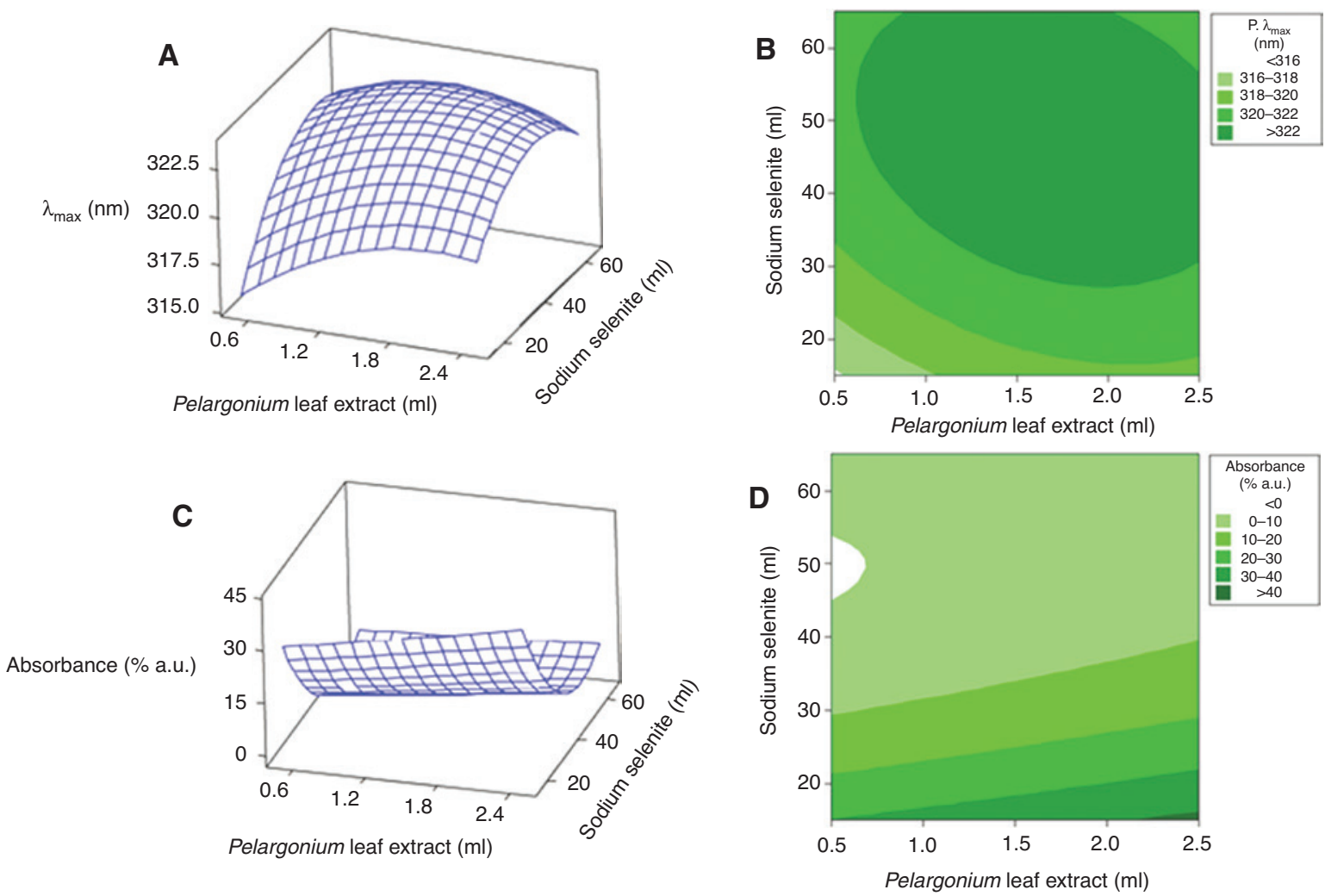

Figure 2: Surface plot (A) and contour plot (B) for $\lambda_{\max }$, and surface plot (C) and contour plot (D) for absorbance of the synthesized selenium nanoparticles (Se NPs) as a function of amounts of $\mathrm{Na}_{2} \mathrm{SeO}_{3}$ solution $(\mathrm{ml})$ and Pelargonium zonale leaves extract (PLE) (ml).

the concentrations of the fabricated NPs drastically increased.

\subsection{Optimization and validation procedures to obtain optimum levels of the synthesis parameters}

The optimum synthesis conditions for fabrication of the inorganic NPs obtained when the process leads to synthesis of the NPs with minimum particle size and higher concentration $[18,24]$ (Figure 3A) indicates the optimum area for the synthesis parameters to produce Se NPs with lower $\lambda_{\max }(\mathrm{nm})$ and higher absorbance (\% a.u.). Numerical multiple optimization also indicated that the strict optimum values of selected fabrication parameters would be attained using $1.48 \mathrm{ml} \mathrm{PLE}$ and $15 \mathrm{ml}$ of $\mathrm{Na}_{2} \mathrm{SeO}_{3}$ solution; the $\lambda_{\max }$ and absorbance of the solution containing Se NPs were 319 (nm) and 35.33 (\% a.u.), respectively. The verification tests for the synthesis of the Se NPs at obtained and predicted optimum conditions resulted in Se NPs with $\lambda_{\max }$ and absorbance of $319 \pm 1(\mathrm{~nm})$ and $34.6 \pm 2$ (\% a.u.), respectively.

\subsection{Characteristics of the fabricated Se NPs at obtained optimum synthesis conditions}

Particle size, PDI and zeta potential values for the formed Se NPs using DLS at obtained optimum conditions were $136 \mathrm{~nm}, 0.321$ and $-24.6 \mathrm{mV}$, respectively. Mixture solutions containing NPs with zeta potential above $+20 \mathrm{mV}$ or below $-20 \mathrm{mV}$ are physically stable [31]. High value of the particle size by DLS is related to the diameter of the synthesized Se NPs and the thick layer of stabilizing compounds which surrounded the Se NPs [32]. The PDI value of the synthesized Se NPs at attained optimum fabrication conditions revealed that the fabricated NPs were moderately polydisperse. The UV-Vis spectra of the formed Se NPs are presented in Figure 3B. As evidently detected in this figure, the formed Se NPs at achieved optimum conditions had $\lambda_{\max }$ of $319 \mathrm{~nm}$. Particle size distribution of the fabricated Se NPs is also shown in Figure 3C.

A typical TEM image gained from the formed Se NPs solution at achieved optimum conditions is displayed in Figure 3D. The TEM image of the Se NPs synthesized revealed the existence of approximately spherical 

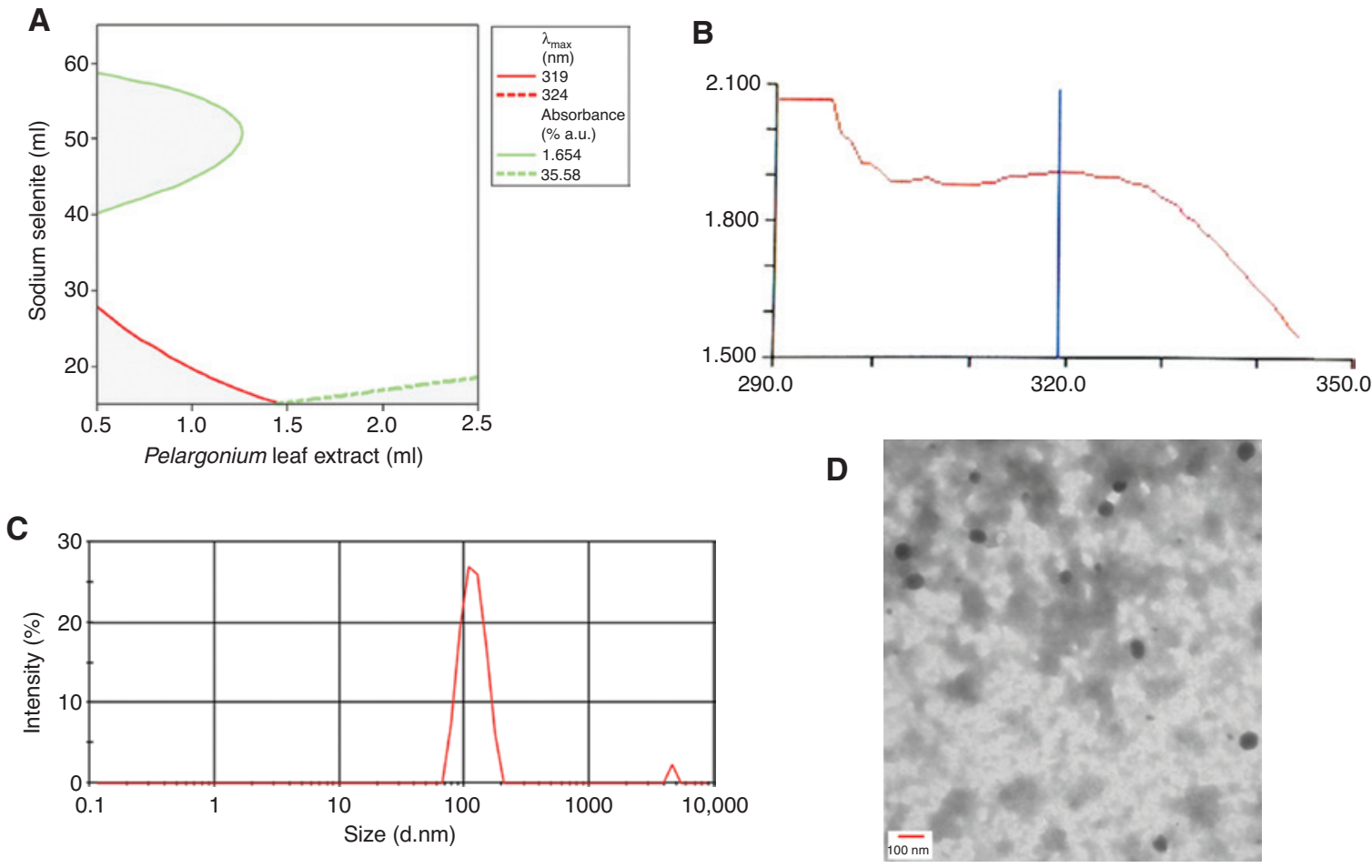

Figure 3: Overlaid contour plot of $\lambda_{\max }$, absorbance with acceptable levels as a function of amount of $\mathrm{Na}_{2} \mathrm{SeO}_{3}$ (ml) and Pelargonium zonale leaves extract (PLE) (ml) (A), UV-Vis spectra (B), particle size distribution (C) and transmission electron microscopy (TEM) image (D) of the synthesized selenium nanoparticles (Se NPs) at obtained optimum conditions.

A

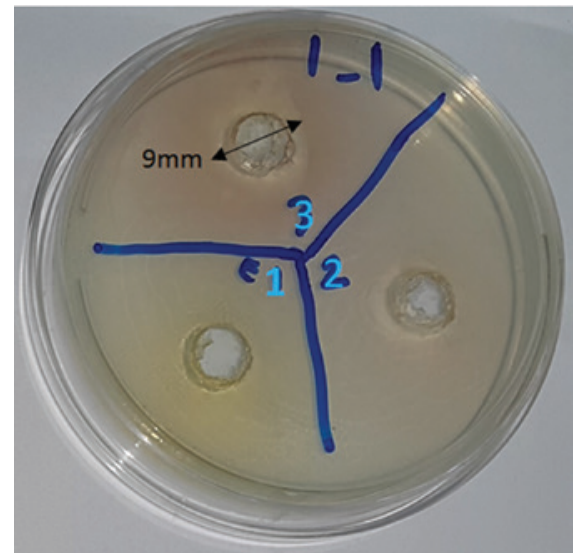

C

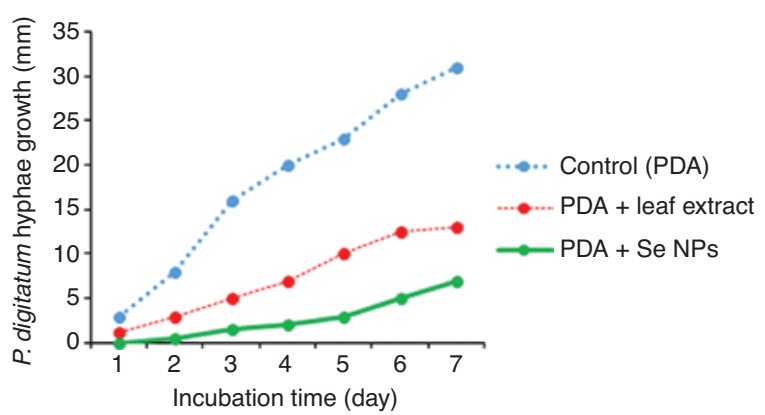

B

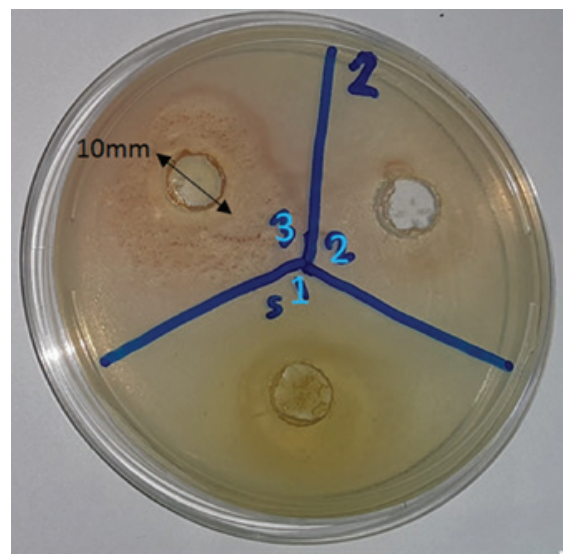

D

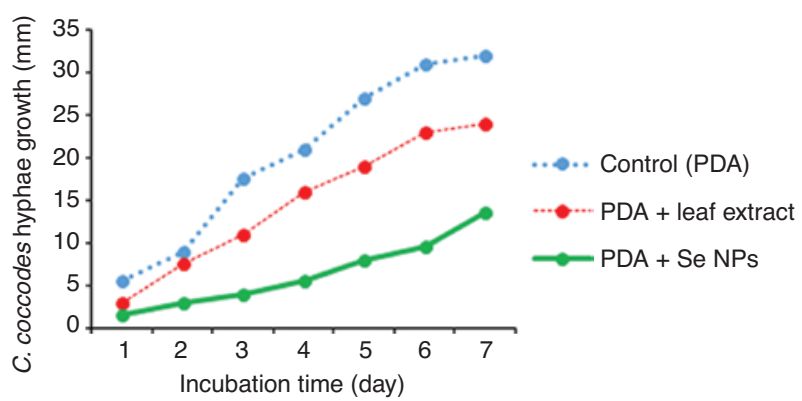

Figure 4: Created zones of inhibition for Pelargonium zonale leaves extract (PLE) (1), $\mathrm{Na}_{2} \mathrm{SeO}_{3}$ (2) and selenium nanoparticles (Se NPs) (3) on the inoculated plates with Escherichia coli (A) and Staphylococcus aureus (B) incubated at $37^{\circ} \mathrm{C}$ for $24 \mathrm{~h}$. Antifungal activity of the synthesized Se NPs at obtained optimum conditions against Penicillium digitatum (C) and Colletotrichum coccodes (D). Data are the mean value of three replicates. 
moderately polydisperse NPs with a particle size range of 40-60 $\mathrm{nm}$. The obtained results were in agreement with findings of other investigators on green synthesis of Se NPs $[6,7,33]$.

\subsection{Antimicrobial activities of the fabricated Se NPs at obtained optimum synthesis conditions}

The antibacterial activities of the synthesized Se NPs as compared to the PLE and $\mathrm{Na}_{2} \mathrm{SeO}_{3}$ solution on the growth of Gram-negative (E. coli) and Gram-positive (S. aureus) bacteria are presented in Figure 4A and B, respectively. As can be seen in these figures, fabricated Se NPs indicated weak antibacterial activities against both bacteria strains. However, the diameter of the inhibition zones around the $S$. aureus $(10 \mathrm{~mm})$ was higher than that around $E$. coli $(9 \mathrm{~mm})$. The obtained results indicated that the PLE and $\mathrm{Na}_{2} \mathrm{SeO}_{3}$ solution had non bactericidal activities toward the studied bacteria strains. The obtained results were in line with findings of other studies which indicated moderate antibacterial activities of the Se NPs toward both Gram-positive and Gram-negative bacteria species $[34,35]$.

The influences of the fabricated Se NPs and PLE on mycelial growth $(\mathrm{mm})$ of $P$. digitatum and $C$. coccodes during the incubation period ( 7 days) are indicated in Figure 4C and D, respectively. As clearly detected in these figures, the synthesized Se NPs had high antifungal activities against both selected fungi strains. However, the fungicidal activity against $P$. digitatum was higher than against $C$. coccodes (Figure $4 \mathrm{C}$ and $\mathrm{D}$ ). The results were in line with the findings of other researches which also revealed higher antifungal activities of the Se NPs [36, 37]. The attained results also specified that the PLE had low antifungal activities toward both studied and selected fungi.

\section{Conclusions}

The present study indicated that Pelargonium zonale leaf extract contains the main bioactive compounds which can be used in synthesizing and stabilizing the Se NPs. Furthermore, RSM could be effectively used to model the synthesis procedure and predict the selected Se NPs attributes influenced by the fabrication variables. The developed synthesis technique in the present study is a clean, fast, cost-effective and one-step synthesis process which can be utilized into the synthesis of other metal and metal oxide NPs.

Acknowledgments: The authors appreciate the financial support of the Iran Nanotechnology Initiatives Council (grant no. 125018).

\section{References}

[1] Preedy VR, Ed., Selenium: Chemistry, Analysis, Function and Effects. Royal Society of Chemistry: Cambridge, 2015.

[2] Chen W, Li Y, Yang S, Yue L, Jiang Q, Xia W. Carbohydr. Polym. 2015, 132, 574-581.

[3] Kong H, Yang J, Zhang Y, Fang Y, Nishinari K, Phillips GO. Int. J. Biol. Macromol. 2014, 65, 155-162.

[4] McClements J, McClements DJ. Crit. Rev. Food Sci. Nutr. 2016, 56, 1334-1362.

[5] Zhou Y, Xu M, Liu Y, Bai Y, Deng Y, Liu J, Chen L. Colloids Surf. B 2016, 144, 118-124.

[6] Chen T, Wong YS, Zheng W, Bai Y, Huang L. Colloids Surf. B 2008, 67, 26-31.

[7] Prasad KS, Patel H, Patel T, Patel K, Selvaraj K. Colloids Surf. B 2013, 103, 261-266.

[8] Fairweather-Tait SJ, Bao Y, Broadley MR, Collings R, Ford D, Hesketh JE, Hurst R. Antioxid. Redox Signaling 2011, 14, 1337-1383.

[9] Ramamurthy C, Sampath KS, Arunkumar P, Kumar MS, Sujatha V, Premkumar K, Thirunavukkarasu C. Bioprocess Biosyst. Eng. 2013, 36, 1131-1139.

[10] Shikuo L, Yuhua S, Anjian X, Xuerong Y, Xiuzhen Z, Liangbao Y, Chuanhao L. Nanotechnology 2007, 18, 405101.

[11] Sharma G, Sharma AR, Bhavesh R, Park J, Ganbold B, Nam JS, Lee SS. Molecules 2014, 19, 2761-2770.

[12] Majid NA, Phang IC, Darnis DS. Environ. Sci. Pollut. Res. 2017, 24, 22827-22838.

[13] Lim TK. In Edible Medicinal and Non Medicinal Plants, Lim TK, Ed., Springer: Netherlands, 2014, Vol. 8, p 72.

[14] Husen A, Siddiqi KS. J. Nanobiotechnol. 2014, 12, 28-38.

[15] Mohammadlou M, Jafarizadeh-Malmiri H, Maghsoudi H. Green Process. Synth. 2017, 6, 31-42.

[16] Ahmadi O, Jafarizadeh-Malmiri H, Jodeiri N. Green Process. Synth. 2018, 7, 231-240.

[17] Eskandari-Nojehdehi M, Jafarizadeh-Malmiri H, Rahbar-Shahrouzi J. Nanotechnol. Rev. 2016, 5, 537-548.

[18] Eskandari-Nojehdehi M, Jafarizadeh-Malmiri H, Jafarizad A. Z. Phys. Chem. 2018, 232, 325-343.

[19] Anu K, Singaravelu G, Murugan K, Benelli G. J. Cluster Sci. 2017, 28, 551-563.

[20] Prasad KS, Selvaraj K. Biol. Trace Elem. Res. 2014, 157, 275-283.

[21] Kapur M, Soni K, Kohli K. Adv. Tech. Biol. Med. 2017, 5, 197-204.

[22] Anil K, Smritilekha B, Man S, Dhananjoy M. Adv. Nat. Sci.: Nanosci. Nanotechnol. 2018, 9, 015004-015014.

[23] Torabfam M, Jafarizadeh-Malmiri H. Green Process. Synth. 2018, 7, 530-537. 
[24] Ghanbari S, Vaghari H, Sayyar Z, Adibpour M, Jafarizadeh-Malmiri H. Green Process. Synth. 2018, 7, 217-224.

[25] Jafari N, Jafarizadeh-Malmiri H, Hamzeh-Mivehroud M, Adibpour M. Green Process. Synth. 2017, 6, 333-340.

[26] Ahdno H, Jafarizadeh-Malmiri H. Food Bioprod. Process. 2017, 101, 193-204.

[27] Anarjan N, Jaberi N, Yeganeh-Zare S, Banafshehchin E, Rahimirad A, Jafarizadeh-Malmiri H. J. Am. Oil Chem. Soc. 2014, 91, 1397-1405.

[28] Amirkhani L, Moghaddas JS, Jafarizadeh-Malmiri H. RSC Adv. 2016, 6, 12676-12687.

[29] Kannan N, Mukunthan KS, Balaji S. Colloids Surf. B 2011, 86, 378-383.

[30] Eskandari-Nojedehi M, Jafarizadeh-Malmiri $\mathrm{H}$, Rahbar-Shahrouzi J. Green Process. Synth. 2018, 7, 38-47.
[31] El-Naggar ME, Shaheen TI, Fouda MM, Hebeish AA. Carbohydr. Polym. 2016, 136, 1128-1136.

[32] Souza TGF, Ciminelli VST, Mohallem NDS. J. Phys.: Conf. Ser. 2016, 733, 012039-012044.

[33] Sowndarya P, Ramkumar G, Shivakumar MS. Artif. Cells Nanomed. Biotechnol. 2017, 45, 1490-1495.

[34] Srivastava N, Mukhopadhyay M. Bioprocess Biosyst. Eng. 2015, 38, 1723-1730.

[35] Shoeibi S, Mashreghi M. J. Trace Elem. Med. Biol. 2017, 39, 135-139.

[36] Shakibaie M, Salari Mohazab N, Ayatollahi Mousavi SA. Jundishapur J. Microbiol. 2015, 8, e26381-e26385.

[37] Shahverdi AR, Fakhimi A, Mosavat G, Jafari-Fesharaki P, Rezaie S, Rezayat SM. World Appl. Sci. J. 2010, 10, 918-922. 